\title{
Finding of the Metric Operator for a Quasi-Hermitian Model
}

\author{
Ebru Ergun \\ Department of Mathematics, Karabuk University, 78050 Karabuk, Turkey \\ Correspondence should be addressed to Ebru Ergun; ebruergun@karabuk.edu.tr
}

Received 18 May 2013; Accepted 12 July 2013

Academic Editor: Stanislav Hencl

Copyright (C) 2013 Ebru Ergun. This is an open access article distributed under the Creative Commons Attribution License, which permits unrestricted use, distribution, and reproduction in any medium, provided the original work is properly cited.

We consider on an appropriate Sobolev space a non-Hermitian Hamiltonian $H_{\alpha, \beta}$ depending on the two complex parameters $\alpha$ and $\beta$ and having real spectrum. We derive a closed formula for a family of the metric operators, which render the Hamiltonian $H_{\alpha, \beta}$ Hermitian. In some particular cases, we calculate the Hermitian counterpart of $H_{\alpha, \beta}$.

\section{Introduction}

In the quasi-Hermitian quantum mechanics introduced in [1] and developed further in [2,3] and other works, an important problem is to construct a new inner product (scalar product) with respect to which the Hamiltonian $H$ (non-Hermitian with respect to the original inner product of the Hilbert space $\mathscr{H}$ ) having real spectrum turns out to be Hermitian (selfadjoint). In other words, one should find an invertible positive bounded operator $\eta_{+}$with bounded inverse, called metric, which fulfills

$$
\left.H^{*} \eta_{+}=\eta_{+} H \quad \text { (i.e., } H^{*}=\eta_{+} H \eta_{+}^{-1}\right)
$$

so that if $\langle\cdot, \cdot\rangle_{+}=\left\langle\eta_{+} \cdot, \cdot\right\rangle$ is a new inner product, then $H$ becomes Hermitian with respect to $\langle\cdot, \cdot\rangle_{+}$, where $H^{*}$ denotes the adjoint operator of $H$ in the Hilbert space $\mathscr{H}$ with the original inner product $\langle\cdot, \cdot\rangle$. If such an operator $\eta_{+}$exists, then the operator $H$ is called quasi-Hermitian.

As is known $[3,4]$, to construct a metric operator $\eta_{+}$, satisfying (1) the formula

$$
\eta_{+}=\sum_{n=1}^{\infty}\left\langle\cdot, \phi_{n}\right\rangle \phi_{n}
$$

can be used provided that the spectrum of $H$ is real and pure discrete (i.e., each point $E$ of the spectrum of $H$ is an eigenvalue of $H$ with finite multiplicity and $E$ is an isolated point of the spectrum) and the eigenvectors of $H$ form a Riesz basis of space $\mathscr{H}$. Here the set $\left\{\phi_{n}\right\}_{n=1}^{\infty}$ is constructed as follows. Let us label the eigenvalues of $H$ with $E_{n}$, and let $\left\{\psi_{n}\right\}_{n=1}^{\infty}$ denote a Riesz basis of $\mathscr{H}$ consisting of the eigenvectors $\psi_{n}$ of $H, H \psi_{n}=E_{n} \psi_{n}$. Then one can construct another Riesz basis $\left\{\phi_{n}\right\}_{n=1}^{\infty}$ of $\mathscr{H}$ that satisfies

$$
H^{*} \phi_{n}=E_{n} \phi_{n}, \quad\left\langle\psi_{n}, \phi_{m}\right\rangle=\delta_{n m}, \quad \sum_{n=1}^{\infty}\left\langle\cdot, \phi_{n}\right\rangle \psi_{n}=I,
$$

where $\delta_{n m}$ is the Kronecker delta and $I$ denotes the identity operator in $\mathscr{H}$. The operator $\eta_{+}$defined by (2) is manifestly positive, and it is boundedly invertible, with the inverse given by

$$
\eta_{+}^{-1}=\sum_{n=1}^{\infty}\left\langle\cdot, \psi_{n}\right\rangle \psi_{n}
$$

We can use (2) to introduce the positive-definite inner product

$$
\langle\cdot, \cdot\rangle_{+}=\left\langle\eta_{+} \cdot, \cdot\right\rangle
$$

and identify the physical Hilbert space $\mathscr{H}_{\text {phys }}$ with the underlying vector space of $\mathscr{H}$ endowed with this inner product. Let $\rho=\sqrt{\eta_{+}}$be the unique positive-definite square root of $\eta_{+}$. Then the operator $\rho$ as a mapping of $\mathscr{H}_{\text {phys }}$ onto $\mathscr{H}$ is a unitary operator and the operator

$$
h=\rho H \rho^{-1}
$$

is Hermitian in the space $\mathscr{H}$ and is called a Hermitian counterpart of $H$. Thus, under a similarity transformation 
implemented by $\rho=\sqrt{\eta_{+}}$, the non-Hermitian Hamiltonian $H$ is equivalent to a Hermitian Hamiltonian $h$, according to (6). The "physical observables" are the Hermitian operators $O$ acting in $\mathscr{H}_{\text {phys. }}$. We can use the unitary-equivalence of $\mathscr{H}_{\text {phys }}$ and $\mathscr{H}$ realized by $\rho$ to construct the physical observables $O$ using those of the conventional quantum mechanics, that is, Hermitian operators $o$ acting in the reference Hilbert space $\mathscr{H}$. This is done according to $O=\rho^{-1} o \rho$ [3]. Since $\rho$ is a unitary operator as a mapping of $\mathscr{H}_{\text {phys }}$ onto $\mathscr{H}, O$ is a Hermitian operator acting in $\mathscr{H}_{\text {phys }}$ if and only if $o$ is a Hermitian operator acting in $\mathscr{H}$.

Since the metric operator $\eta_{+}$is of central importance, many attempts have been made to construct it when given only a non-Hermitian Hamiltonian. In general, the construction of a metric operator is a difficult problem, and therefore most of the available formulas for $\eta_{+}$are just approximative, expressed as leading terms of perturbation series [3,5]. So far, one has only succeeded to compute exact expressions for the metric and Hermitian counterparts in very few cases of nonHermitian Hamiltonians (for some of them, see [6-12]).

In the present paper, we deal with the eigenvalue problem

$$
\begin{gathered}
-\psi^{\prime \prime}(x)=\lambda \psi(x), \quad x \in[-1,0) \cup(0,1], \\
\psi\left(0^{-}\right)=\alpha \psi\left(0^{+}\right), \quad \psi^{\prime}\left(0^{-}\right)=\beta \psi^{\prime}\left(0^{+}\right), \\
\psi^{\prime}(-1)=\psi^{\prime}(1)=0,
\end{gathered}
$$

where $\lambda$ is a spectral parameter, $\alpha$ and $\beta$ are fixed nonzero complex numbers, and $\psi$ is a function from the Sobolev space $W^{2,2}([-1,0) \cup(0,1])$. The space $W^{2,2}([-1,0) \cup(0,1])$ consists of all complex-valued functions $\psi \in L^{2}(-1,1)$ differentiable on each of the intervals $[-1,0)$ and $(0,1]$ with the derivative $\psi^{\prime}$ absolutely continuous on each closed subinterval of the intervals $[-1,0)$ and $(0,1]$ (therefore there exists the second derivative $\psi^{\prime \prime}$ almost every where because any function which is absolutely continuous on an interval is differentiable almost everywhere on that interval) and such that

$$
\int_{-1}^{0}\left|\psi^{\prime \prime}(x)\right|^{2} d x<\infty, \quad \int_{0}^{1}\left|\psi^{\prime \prime}(x)\right|^{2} d x<\infty .
$$

It can easily be shown (see [11]) that for any function $\psi \in W^{2,2}([-1,0) \cup(0,1])$, there exist the finite limit values $\psi\left(0^{-}\right), \psi^{\prime}\left(0^{-}\right), \psi\left(0^{+}\right)$, and $\psi^{\prime}\left(0^{+}\right)$. The conditions in (8) are called the transition conditions (matching conditions or impulse conditions), while the conditions in (9) are called the boundary conditions.

Problem (7), (8), and (9) with the Dirichlet boundary conditions

$$
\psi(-1)=\psi(1)=0
$$

instead of the Neumann boundary conditions (9) was before investigated by the author in $[11,12]$.

The model (8) in the PT-symmetric case $|\alpha|=|\beta|=$ 1 had been investigated for the first time by Siegl in [9] where explicit formulas for the metric and its square root are presented.

This paper is organized as follows. In Section 2, we introduce the Hamiltonian $H_{\alpha, \beta}$ corresponding to the eigenvalue problem (7), (8), (9) and formulate the main results of the paper in the form of three theorems. The first two of them determine the regions in the space of the complex parameters $\alpha$ and $\beta$ where $H_{\alpha, \beta}$ is (i) Hermitian, (ii) $P T$-symmetric, while the third theorem presents a closed formula for a family of the metric operators depending on two arbitrary real positive parameters when $H_{\alpha, \beta}$ is non-Hermitian having real spectrum. In Section 3, the adjoint operator of the Hamiltonian $H_{\alpha, \beta}$ is described. Section 4 provides the spectral analysis of the Hamiltonian $H_{\alpha, \beta}$ and its adjoint calculating explicitly the eigenvalues and eigenfunctions of these operators. Section 5 contains a proof of Theorem 3 deriving a closed formula for a family of the metric operators. In Section 6, we calculate a Hermitian counterpart of $H_{\alpha, \beta}$ in two particular cases. Finally, in Section 7, we make a short conclusion.

\section{Main Results}

To introduce the Hamiltonian corresponding to the eigenvalue problem (7), (8), and (9), consider the standard Hilbert space $\mathscr{H}=L^{2}(-1,1)$ with the usual inner product

$$
\langle\psi, \phi\rangle=\int_{-1}^{1} \psi(x) \bar{\phi}(x) d x,
$$

where the bar over a function (or over a number) denotes the complex conjugate. Note that the Sobolev space $W^{2,2}([-1,0) \cup$ $(0,1])$ forms a subspace of $L^{2}(-1,1)$.

In the Hilbert space $\mathscr{H}=L^{2}(-1,1)$, we consider the Hamiltonian $H_{\alpha, \beta}$ defined as follows. The domain of definition $D\left(H_{\alpha, \beta}\right)$ of the operator $H_{\alpha, \beta}$ consists of all functions $\psi \in W^{2,2}([-1,0) \cup(0,1])$ satisfying the transition conditions (8) and the boundary conditions (9). On the functions $\psi \in$ $D\left(H_{\alpha, \beta}\right)$, the Hamiltonian $H_{\alpha, \beta}$ is given by

$$
\left(H_{\alpha, \beta} \psi\right)(x)=-\psi^{\prime \prime}(x), \quad x \in[-1,0) \cup(0,1] \text {. }
$$

Let $P$ and $T$ be the parity and the time reversal operators defined by

$$
(P \psi)(x)=\psi(-x), \quad(T \psi)(x)=\bar{\psi}(x) .
$$

The Hamiltonian $H_{\alpha, \beta}$ is called $P T$-symmetric if for every $\psi \epsilon$ $D\left(H_{\alpha, \beta}\right)$, we have that $P T \psi \in D\left(H_{\alpha, \beta}\right)$ and

$$
H_{\alpha, \beta} P T \psi=P T H_{\alpha, \beta} \psi
$$

Main results of the present paper can be formulated as the following theorems.

Theorem 1. The operator $H_{\alpha, \beta}$ is Hermitian if and only if $\alpha \bar{\beta}=$ 1.

Theorem 2. The operator $H_{\alpha, \beta}$ is PT-symmetric if and only if $|\alpha|=1$ and $|\beta|=1$.

Theorem 3. Assume that the complex numbers $\alpha$ and $\beta$ are nonzero. If $\alpha+\beta=0$, then any complex number is an 
eigenvalue of the operator $H_{\alpha, \beta}$, but if $\alpha+\beta \neq 0$, then the spectrum of $H_{\alpha, \beta}$ is real and consists of the simple eigenvalues

$$
\lambda_{n}=\left(\frac{n}{2} \pi\right)^{2}, \quad \text { where } n=0,1,2, \ldots
$$

Furthermore, let $\alpha+\beta \neq 0$ and $\eta_{+}$be a linear operator defined on $\mathscr{H}$ by

$$
\begin{aligned}
& \left(\eta_{+} \psi\right)(x) \\
& =\frac{1}{2}\left\{\begin{array}{r}
(A+B) \psi(x)+(A \beta-B \alpha) \psi(-x) \\
\text { if } x \in[-1,0), \\
\left(A|\beta|^{2}+B|\alpha|^{2}\right) \psi(x)+(A \bar{\beta}-B \bar{\alpha}) \psi(-x) \\
\text { if } x \in(0,1],
\end{array}\right.
\end{aligned}
$$

where $A$ and $B$ are arbitrary positive real numbers. Then $\eta_{+}$is an invertible positive bounded operator with bounded inverse and satisfies

$$
\begin{gathered}
\forall \psi \in D\left(H_{\alpha, \beta}\right) \Longrightarrow \eta_{+} \psi \in D\left(H_{\alpha, \beta}^{*}\right), \\
H_{\alpha, \beta}^{*} \eta_{+} \psi=\eta_{+} H_{\alpha, \beta} \psi
\end{gathered}
$$

so that the Hamiltonian $\mathrm{H}_{\alpha, \beta}$ is quasi-Hermitian in this case and $\eta_{+}$is a metric operator for it.

\section{The Adjoint of the Hamiltonian}

Theorem 4. For any nonzero complex numbers $\alpha$ and $\beta$ such that $\alpha+\beta \neq 0$, the relation

$$
H_{\alpha, \beta}^{*}=H_{1 / \bar{\beta}, 1 / \bar{\alpha}}
$$

holds. Therefore, the domain of definition $D\left(H_{\alpha, \beta}^{*}\right)$ of $H_{\alpha, \beta}^{*}$ consists of all functions $\phi \in W^{2,2}([-1,0) \cup(0,1])$ satisfying the transition conditions

$$
\phi\left(0^{-}\right)=\frac{1}{\bar{\beta}} \phi\left(0^{+}\right), \quad \phi^{\prime}\left(0^{-}\right)=\frac{1}{\bar{\alpha}} \phi^{\prime}\left(0^{+}\right)
$$

and the boundary conditions

$$
\phi^{\prime}(-1)=\phi^{\prime}(1)=0,
$$

and on such functions $\phi$,

$$
\left(H_{\alpha, \beta}^{*} \phi\right)(x)=-\phi^{\prime \prime}(x), \quad x \in[-1,0) \cup(0,1] .
$$

Proof. Recall that by definition of the adjoint operator, the domain of definition $D\left(H_{\alpha, \beta}^{*}\right)$ of $H_{\alpha, \beta}^{*}$ consists of those functions $\phi$ in $L^{2}(-1,1)$ such that

$$
\left\langle H_{\alpha, \beta} \psi, \phi\right\rangle=\langle\psi, f\rangle
$$

for all $\psi$ in $D\left(H_{\alpha, \beta}\right)$ and some $f$ in $L^{2}(-1,1)$ depending on $\phi$. Since $D\left(H_{\alpha, \beta}\right)$ is dense in $L^{2}(-1,1), f$ is uniquely determined by $\phi$ and, by definition, $H_{\alpha, \beta}^{*} \phi=f$.
The inclusion

$$
D\left(H_{1 / \bar{\beta}, 1 / \bar{\alpha}}\right) \subset D\left(H_{\alpha, \beta}^{*}\right)
$$

is easy to see. Indeed, take any $\phi \in D\left(H_{1 / \bar{\beta}, 1 / \bar{\alpha}}\right)$. Hence, $\phi \in$ $W^{2,2}([-1,0) \cup(0,1])$ and satisfies conditions (20) and (21). Then for every $\psi \in D\left(H_{\alpha, \beta}\right)$ we have, integrating by parts twice and taking into account that $\psi$ satisfies (8) and (9),

$$
\begin{aligned}
& \left\langle H_{\alpha, \beta} \psi, \phi\right\rangle \\
& =-\int_{-1}^{1} \psi^{\prime \prime}(x) \bar{\phi}(x) d x \\
& =-\int_{-1}^{0} \psi^{\prime \prime}(x) \bar{\phi}(x) d x-\int_{0}^{1} \psi^{\prime \prime}(x) \bar{\phi}(x) d x \\
& =-\left.\psi^{\prime}(x) \bar{\phi}(x)\right|_{-1} ^{0^{-}}+\left.\psi(x) \bar{\phi}^{\prime}(x)\right|_{-1} ^{0^{-}} \\
& -\int_{-1}^{0} \psi(x) \bar{\phi}^{\prime \prime}(x) d x \\
& -\left.\psi^{\prime}(x) \bar{\phi}(x)\right|_{0^{+}} ^{1}+\left.\psi(x) \bar{\phi}^{\prime}(x)\right|_{0^{+}} ^{1} \\
& -\int_{0}^{1} \psi(x) \bar{\phi}^{\prime \prime}(x) d x \\
& =\left[-\beta \bar{\phi}\left(0^{-}\right)+\bar{\phi}\left(0^{+}\right)\right] \psi^{\prime}\left(0^{+}\right) \\
& +\left[\alpha \bar{\phi}^{\prime}\left(0^{-}\right)-\bar{\phi}^{\prime}\left(0^{+}\right)\right] \psi\left(0^{+}\right) \\
& +\bar{\phi}^{\prime}(-1) \psi(-1)-\bar{\phi}^{\prime}(1) \psi(1) \\
& -\int_{-1}^{1} \psi(x) \bar{\phi}^{\prime \prime}(x) d x \\
& =-\int_{-1}^{1} \psi(x) \bar{\phi}^{\prime \prime}(x) d x \text {. }
\end{aligned}
$$

The sum of terms outside the integrals became zero because of the fact that $\phi$ satisfies (20) and (21). This shows that $\phi$ belongs to $D\left(H_{\alpha, \beta}^{*}\right)$ (therefore (24) holds), and on such elements $\phi$, we have equality (22). Note that in this part, we did not use the condition $\alpha+\beta \neq 0$.

To complete the proof of the theorem, where this is the major part of the proof, we should show that the opposite of (24) also holds and that on any $\phi \in D\left(H_{\alpha, \beta}^{*}\right)$ we have equality (22). This can be done similarly to that given in [11] in the proof of Proposition 4.

From Theorem 4, we get Theorem 1 as a corollary under the condition $\alpha+\beta \neq 0$. On the other hand, if $\alpha+\beta=0$, the operator $H_{\alpha, \beta}$ cannot be Hermitian because in this case $H_{\alpha, \beta}$ has nonreal eigenvalues (see Theorem 5(ii)).

Theorem 2 follows exactly in the same way as in [11, Section 4].

Applying Theorem 4 to the operator $H_{1 / \bar{\beta}, 1 / \bar{\alpha}}$, we get that $H_{1 / \bar{\beta}, 1 / \bar{\alpha}}^{*}=H_{\alpha, \beta}$. Therefore, for any nonzero complex numbers $\alpha$ and $\beta$ with $\alpha+\beta \neq 0$, the operator $H_{\alpha, \beta}$ is closed because, as 
is known (and it can easily be seen), the adjoint of any densely defined linear operator is a closed operator.

\section{Spectral Analysis of the Hamiltonian and Its Adjoint}

In this section, we study the spectrum of the operator $H_{\alpha, \beta}$.

Theorem 5. Let $\alpha$ and $\beta$ be nonzero complex numbers. Then, one has the following.

(i) If $\alpha+\beta \neq 0$, then the spectrum of the operator $H_{\alpha, \beta}$ is real and consists of the (simple) eigenvalues

$$
\lambda_{n}=\left(\frac{n}{2} \pi\right)^{2}, \quad \text { where } n=0,1,2, \ldots
$$

The corresponding eigenfunctions $\psi_{n}$ are found by

$$
\begin{gathered}
\psi_{2 j}(x)=c_{2 j} \begin{cases}\alpha \cos j \pi x & \text { if } x \in[-1,0), \\
\cos j \pi x & \text { if } x \in(0,1],\end{cases} \\
\psi_{2 j+1}(x)=c_{2 j+1} \begin{cases}\beta \sin \left(j+\frac{1}{2}\right) \pi x & \text { if } x \in[-1,0), \\
\sin \left(j+\frac{1}{2}\right) \pi x & \text { if } x \in(0,1],\end{cases}
\end{gathered}
$$

for $j=0,1,2, \ldots$, where $c_{n}$ are nonzero complex constants which may depend on $\alpha$ and $\beta$.

(ii) If $\alpha+\beta=0$, then any complex number $\lambda=k^{2}(k \in$ $\mathbb{C})$ is an eigenvalue of the operator $H_{\alpha, \beta}$ with the corresponding eigenfunction

$$
\psi(x)=c \begin{cases}\alpha \cos k(x+1) & \text { if } x \in[-1,0), \\ \cos k(x-1) & \text { if } x \in(0,1],\end{cases}
$$

where $c$ is an arbitrary nonzero complex constant which may depend on $\lambda$.

This theorem is elementary and can be proved as in [11]. Note that it can also be verified directly by substituting (27) and (28) in (7), (8), and (9).

From Theorems 4 and 5, it follows that if $\alpha+$ $\beta \neq 0(\alpha \neq 0, \beta \neq 0)$ the spectrum of the adjoint operator $H_{\alpha, \beta}^{*}$ coincides with the set of eigenvalues $\lambda_{n}$ given in (26), and the corresponding eigenfunctions $\phi_{n}$ are given by

$$
\begin{gathered}
\phi_{2 j}(x)=d_{2 j} \begin{cases}\frac{1}{\bar{\beta}} \cos j \pi x & \text { if } x \in[-1,0), \\
\cos j \pi x & \text { if } x \in(0,1],\end{cases} \\
\phi_{2 j+1}(x)=d_{2 j+1} \begin{cases}\frac{1}{\bar{\alpha}} \sin \left(j+\frac{1}{2}\right) \pi x & \text { if } x \in[-1,0), \\
\sin \left(j+\frac{1}{2}\right) \pi x & \text { if } x \in(0,1],\end{cases}
\end{gathered}
$$

for $j=0,1,2, \ldots$, where $d_{n}$ are nonzero complex constants which may depend on $\alpha$ and $\beta$.

From

$$
H_{\alpha, \beta} \psi_{n}=\lambda_{n} \psi_{n}, \quad H_{\alpha, \beta}^{*} \phi_{m}=\lambda_{m} \phi_{m}
$$

it follows, by a standard way, that $\psi_{n}$ and $\phi_{m}$ are orthogonal to each other provided $n \neq m$. We can normalize the eigenfunctions appropriately to get

$$
\left\langle\psi_{n}, \phi_{m}\right\rangle=\delta_{n m} \quad(n, m=0,1,2, \ldots),
$$

where $\delta_{n m}$ is the Kronecker delta. Namely, taking into account

$$
\int_{0}^{1} \cos ^{2} j \pi x d x=\int_{0}^{1} \sin ^{2}\left(j+\frac{1}{2}\right) \pi x d x=\frac{1}{2},
$$

it is easy to see that (31) follows by choosing the coefficients $c_{n}$ and $d_{n}$ in (27) and (29) according to the equations

$$
\begin{gathered}
1=\frac{\alpha+\beta}{2 \beta} c_{2 j} \bar{d}_{2 j}, \\
1=\frac{\alpha+\beta}{2 \alpha} c_{2 j+1} \bar{d}_{2 j+1} \quad(j=0,1,2, \ldots) .
\end{gathered}
$$

These equations can obviously be satisfied. We choose the coefficient $d_{n}$ by the requirement

$$
d_{2 j}=a \bar{\beta}, \quad d_{2 j+1}=b \bar{\alpha} \quad(j=0,1,2, \ldots),
$$

where $a$ and $b$ are arbitrary nonzero complex numbers, determining $c_{n}$ by the equations in (33):

$$
c_{2 j}=\frac{2 \bar{a}}{\alpha+\beta}, \quad c_{2 j+1}=\frac{2 \bar{b}}{\alpha+\beta} \quad(j=0,1,2, \ldots) .
$$

Then we have

$$
\begin{aligned}
& \psi_{2 j}(x)=\frac{\bar{a} \sqrt{2}}{\alpha+\beta} \begin{cases}\alpha u_{j}(x) & \text { if } x \in[-1,0), \\
u_{j}(x) & \text { if } x \in(0,1],\end{cases} \\
& \psi_{2 j+1}(x)=\frac{\bar{b} \sqrt{2}}{\alpha+\beta} \begin{cases}\beta v_{j}(x) & \text { if } x \in[-1,0), \\
v_{j}(x) & \text { if } x \in(0,1],\end{cases} \\
& \phi_{2 j}(x)=\frac{a}{\sqrt{2}} \begin{cases}u_{j}(x) & \text { if } x \in[-1,0), \\
\bar{\beta} u_{j}(x) & \text { if } x \in(0,1],\end{cases} \\
& \phi_{2 j+1}(x)=\frac{b}{\sqrt{2}} \begin{cases}v_{j}(x) & \text { if } x \in[-1,0), \\
\bar{\alpha} v_{j}(x) & \text { if } x \in(0,1],\end{cases}
\end{aligned}
$$

for $j=0,1,2, \ldots$, where

$$
\begin{gathered}
u_{j}(x)=\sqrt{2} \cos j \pi x, \\
v_{j}(x)=\sqrt{2} \sin \left(j+\frac{1}{2}\right) \pi x \quad(j=0,1,2, \ldots) .
\end{gathered}
$$

Note that $\left\{u_{j}\right\}_{j=0}^{\infty}$ forms a set of orthonormal in $L^{2}(0,1)$ eigenfunctions of the Hermitian Sturm-Liouville eigenvalue problem

$$
\begin{gathered}
-u^{\prime \prime}(x)=\lambda u(x), \quad x \in[0,1], \\
u^{\prime}(0)=u^{\prime}(1)=0,
\end{gathered}
$$


and $\left\{v_{j}\right\}_{j=0}^{\infty}$ forms a set of orthonormal in $L^{2}(0,1)$ eigenfunctions of the Hermitian Sturm-Liouville eigenvalue problem

$$
\begin{gathered}
-v^{\prime \prime}(x)=\lambda v(x), \quad x \in[0,1], \\
v(0)=v^{\prime}(1)=0 .
\end{gathered}
$$

As is well known, the sets $\left\{u_{j}\right\}_{j=0}^{\infty}$ and $\left\{v_{j}\right\}_{j=0}^{\infty}$ form complete orthonormal families (orthonormal bases) in the Hilbert space $L^{2}(0,1)$. In particular, we have the expansions

$$
\begin{aligned}
& f(x)=\sum_{j=0}^{\infty} u_{j}(x) \int_{0}^{1} f(t) u_{j}(t) d t \\
& f(x)=\sum_{j=0}^{\infty} v_{j}(x) \int_{0}^{1} f(t) v_{j}(t) d t
\end{aligned}
$$

and the Parseval equalities

$$
\begin{aligned}
\int_{0}^{1}|f(x)|^{2} d x & =\sum_{j=0}^{\infty}\left|\int_{0}^{1} f(t) u_{j}(t) d t\right|^{2} \\
& =\sum_{j=0}^{\infty}\left|\int_{0}^{1} f(t) v_{j}(t) d t\right|^{2}
\end{aligned}
$$

for every $f \in L^{2}(0,1)$.

Let us calculate the resolvent of the operator $H_{\alpha, \beta}$, where $\alpha$ and $\beta$ are nonzero complex numbers with $\alpha+\beta \neq 0$. To this end, we introduce the solutions $\varphi(x, \lambda)$ and $\theta(x, \lambda)$ of (7) satisfying the transition conditions (8) and the initial conditions

$$
\begin{array}{cl}
\varphi(-1, \lambda)=1, & \varphi^{\prime}(-1, \lambda)=0, \\
\theta(1, \lambda)=1, & \theta^{\prime}(1, \lambda)=0,
\end{array}
$$

respectively. Note that $\varphi(x, \lambda)$ satisfies the boundary condition in (9) at $x=-1$ and $\theta(x, \lambda)$ at $x=1$. Putting $\lambda=k^{2}$, we can find these solutions explicitly as follows:

$$
\begin{aligned}
& \varphi(x, \lambda)= \begin{cases}\cos k(x+1), & x \in[-1,0), \\
\frac{1}{\alpha} \cos k \cos k x-\frac{1}{\beta} \sin k \sin k x, & x \in(0,1],\end{cases} \\
& \theta(x, \lambda)= \begin{cases}\alpha \cos k \cos k x+\beta \sin k \sin k x, & x \in[-1,0), \\
\cos k(x-1), & x \in(0,1]\end{cases}
\end{aligned}
$$

The Wronskian of these solutions is

$$
\begin{aligned}
W_{x}(\varphi, \theta) & =\varphi(x, \lambda) \theta^{\prime}(x, \lambda)-\varphi^{\prime}(x, \lambda) \theta(x, \lambda) \\
& = \begin{cases}\frac{1}{2}(\alpha+\beta) k \sin 2 k, & x \in[-1,0), \\
\frac{1}{2} \frac{\alpha+\beta}{\alpha \beta} k \sin 2 k, & x \in(0,1] .\end{cases}
\end{aligned}
$$

Therefore, by the standard Green function approach, the resolvent $R_{\lambda}=\left(H_{\alpha, \beta}-\lambda I\right)^{-1}$ of the operator $H_{\alpha, \beta}$ has the form, for $\lambda \neq \lambda_{n}(n=0,1,2, \ldots)$,

$$
R_{\lambda} f(x)=\int_{-1}^{1} G(x, y, \lambda) f(y) d y, \quad x \in[-1,0) \cup(0,1],
$$

where the function $G(x, y, \lambda)$ is defined for $x, y \in[-1,0) \cup$ $(0,1]$ by the formula

$$
\begin{aligned}
G(x, y, \lambda)= & -\frac{1}{W_{y}(\varphi, \theta)} \\
& \times \begin{cases}\varphi(x, \lambda) \theta(y, \lambda), & -1 \leq x \leq y \leq 1, \\
\varphi(y, \lambda) \theta(x, \lambda), & -1 \leq y \leq x \leq 1 .\end{cases}
\end{aligned}
$$

It follows from the explicit form of $G(x, y, \lambda)$ that

$$
\int_{-1}^{1} \int_{-1}^{1}|G(x, y, \lambda)|^{2} d x d y<\infty
$$

Therefore, the resolvent of $H_{\alpha, \beta}$ is a compact operator (moreover, a Hilbert-Schmidt operator). This implies that the spectrum of $H_{\alpha, \beta}$ is purely discrete and consists of the eigenvalues $\lambda_{n}=(\pi n / 2)^{2}, n=0,1,2, \ldots$.

\section{Proof of Theorem 3}

To use formula (2) for constructing a metric operator for our Hamiltonian $H_{\alpha, \beta}$, we have to show that the set $\left\{\psi_{n}\right\}_{n=0}^{\infty}$ of eigenfunctions $\psi_{n}$ of $H_{\alpha, \beta}$ forms a Riesz basis of the Hilbert space $\mathscr{H}=L^{2}(-1,1)$. For this purpose, we will use the following result (see [13, Chapter 6, Theorem 2.1]). Suppose a sequence $\left\{\psi_{n}\right\}_{n=0}^{\infty}$ is complete in the Hilbert space $\mathscr{H}$, there corresponds to it a complete biorthogonal sequence $\left\{\phi_{n}\right\}_{n=0}^{\infty}$, and for any $f \in \mathscr{H}$, one has

$$
\sum_{n=0}^{\infty}\left|\left\langle f, \psi_{n}\right\rangle\right|^{2}<\infty, \quad \sum_{n=0}^{\infty}\left|\left\langle f, \phi_{n}\right\rangle\right|^{2}<\infty .
$$

Then the sequence $\left\{\psi_{n}\right\}_{n=0}^{\infty}$ forms a Riesz basis of the space $\mathscr{H}$.

To prove the completeness in $L^{2}(-1,1)$ of the functions $\psi_{n}$ defined by (27), assume that an element $\psi \in L^{2}(-1,1)$ is orthogonal to all the functions $\psi_{n}$ so that we have

$$
\begin{gathered}
\bar{\alpha} \int_{-1}^{0} \psi(x) \cos j \pi x d x+\int_{0}^{1} \psi(x) \cos j \pi x d x=0 \\
\bar{\beta} \int_{-1}^{0} \psi(x) \sin \left(j+\frac{1}{2}\right) \pi x d x \\
+\int_{0}^{1} \psi(x) \sin \left(j+\frac{1}{2}\right) \pi x d x=0
\end{gathered}
$$

for all $j=0,1,2, \ldots$. We have to show that then $\psi=0$. From (52), we get

$$
\begin{gathered}
\int_{0}^{1}[\bar{\alpha} \psi(-x)+\psi(x)] \cos j \pi x d x d x=0, \\
\int_{0}^{1}[-\bar{\beta} \psi(-x)+\psi(x)] \sin \left(j+\frac{1}{2}\right) \pi x d x=0,
\end{gathered}
$$


for all $j=0,1,2, \ldots$. Since the sets $\{\cos j \pi x\}_{j=0}^{\infty}$ and $\{\sin (j+$ $1 / 2) \pi x\}_{j=0}^{\infty}$ form complete orthogonal families (orthogonal bases) in the Hilbert space $L^{2}(0,1)$ as the sets of eigenfunctions of the Hermitian Sturm-Liouville eigenvalue problems (41) and (42), respectively, we conclude from the last equations that

$$
\begin{gathered}
\bar{\alpha} \psi(-x)+\psi(x)=0, \\
-\bar{\beta} \psi(-x)+\psi(x)=0, \quad x \in[0,1] .
\end{gathered}
$$

Hence by the condition $\alpha+\beta \neq 0$, we get $\psi(-x)=\psi(x)=$ $0, x \in[0,1]$. Therefore, $\psi(x)=0, x \in[-1,1]$, and the desired completeness is shown.

We can show similarly that the system $\left\{\phi_{n}\right\}_{n=0}^{\infty}$ also is complete in $L^{2}(-1,1)$. Next, $\left\{\psi_{n}\right\}_{n=0}^{\infty}$ and $\left\{\phi_{n}\right\}_{n=0}^{\infty}$ are biorthogonal by (31), and the conditions in (51) are fulfilled for the functions $\psi_{n}$ and $\phi_{n}$ defined by (36)-(39) in virtue of (44).

Now, we are in a position to use formula (2) for calculating the metric operator $\eta_{+}$for the Hamiltonian $H_{\alpha, \beta}$. Using formulas (38) and (39) for $\phi_{n}$, we find that for any $\psi \in$ $L^{2}(-1,1)$,

$$
\begin{aligned}
& \left(\eta_{+} \psi\right)(x) \\
& =\sum_{n=0}^{\infty}\left\langle\psi, \phi_{n}\right\rangle \phi_{n} \\
& =\sum_{j=0}^{\infty}\left\langle\psi, \phi_{2 j}\right\rangle \phi_{2 j}+\sum_{j=0}^{\infty}\left\langle\psi, \phi_{2 j+1}\right\rangle \phi_{2 j+1} \\
& =\frac{\bar{a}}{\sqrt{2}} \sum_{j=0}^{\infty}\left\{\int_{-1}^{0} \psi(t) u_{j}(t) d t+\beta \int_{0}^{1} \psi(t) u_{j}(t) d t\right\} \phi_{2 j}(x) \\
& +\frac{\bar{b}}{\sqrt{2}} \sum_{j=0}^{\infty}\left\{\int_{-1}^{0} \psi(t) v_{j}(t) d t\right. \\
& \left.+\alpha \int_{0}^{1} \psi(t) v_{j}(t) d t\right\} \phi_{2 j+1}(x) .
\end{aligned}
$$

Hence, if $x \in[-1,0)$, then

$$
\begin{aligned}
& \left(\eta_{+} \psi\right)(x) \\
& =\frac{|a|^{2}}{2} \sum_{j=0}^{\infty}\left\{\int_{-1}^{0} \psi(t) u_{j}(t) d t+\beta \int_{0}^{1} \psi(t) u_{j}(t) d t\right\} u_{j}(x) \\
& +\frac{|b|^{2}}{2} \sum_{j=0}^{\infty}\left\{\int_{-1}^{0} \psi(t) v_{j}(t) d t\right. \\
& \left.+\alpha \int_{0}^{1} \psi(t) v_{j}(t) d t\right\} v_{j}(x),
\end{aligned}
$$

and if $x \in(0,1]$, then

$$
\begin{aligned}
& \left(\eta_{+} \psi\right)(x) \\
& =\frac{|a|^{2}}{2} \sum_{j=0}^{\infty}\left\{\int_{-1}^{0} \psi(t) u_{j}(t) d t\right. \\
& \left.+\beta \int_{0}^{1} \psi(t) u_{j}(t) d t\right\} \bar{\beta} u_{j}(x) \\
& +\frac{|b|^{2}}{2} \sum_{j=0}^{\infty}\left\{\int_{-1}^{0} \psi(t) v_{j}(t) d t\right. \\
& \left.+\alpha \int_{0}^{1} \psi(t) v_{j}(t) d t\right\} \bar{\alpha} v_{j}(x) .
\end{aligned}
$$

Next, using the expansion formulas given in (43) and taking into account that $u_{j}(-x)=u_{j}(x), v_{j}(-x)=-v_{j}(x)$, we have the following.

$$
\text { If } x \in[-1,0) \text {, then }
$$

$$
\begin{aligned}
& \sum_{j=0}^{\infty} u_{j}(x) \int_{-1}^{0} \psi(t) u_{j}(t) d t \\
& =\sum_{j=0}^{\infty} u_{j}(-x) \int_{0}^{1} \psi(-t) u_{j}(t) d t=\psi(x), \\
& \sum_{j=0}^{\infty} u_{j}(x) \int_{0}^{1} \psi(t) u_{j}(t) d t \\
& =\sum_{j=0}^{\infty} u_{j}(-x) \int_{0}^{1} \psi(t) u_{j}(t) d t=\psi(-x), \\
& \sum_{j=0}^{\infty} v_{j}(x) \int_{-1}^{0} \psi(t) v_{j}(t) d t \\
& =\sum_{j=0}^{\infty} v_{j}(-x) \int_{0}^{1} \psi(-t) v_{j}(t) d t=\psi(x), \\
& \sum_{j=0}^{\infty} v_{j}(x) \int_{0}^{1} \psi(t) v_{j}(t) d t \\
& =-\sum_{j=0}^{\infty} v_{j}(-x) \int_{0}^{1} \psi(t) v_{j}(t) d t=-\psi(-x) .
\end{aligned}
$$

If $x \in(0,1]$, then

$$
\begin{aligned}
& \sum_{j=0}^{\infty} u_{j}(x) \int_{-1}^{0} \psi(t) u_{j}(t) d t \\
& =\sum_{j=0}^{\infty} u_{j}(x) \int_{0}^{1} \psi(-t) u_{j}(t) d t=\psi(-x), \\
& \sum_{j=0}^{\infty} u_{j}(x) \int_{0}^{1} \psi(t) u_{j}(t) d t=\psi(x),
\end{aligned}
$$




$$
\begin{aligned}
& \sum_{j=0}^{\infty} v_{j}(x) \int_{0}^{1} \psi(t) v_{j}(t) d t=\psi(x) \\
& \sum_{j=0}^{\infty} v_{j}(x) \int_{-1}^{0} \psi(t) v_{j}(t) d t \\
& =-\sum_{j=0}^{\infty} v_{j}(x) \int_{0}^{1} \psi(-t) v_{j}(t) d t=-\psi(-x) .
\end{aligned}
$$

Substituting these in (56) and (57), we arrive at formula (17) with $A=|a|^{2}$ and $B=|b|^{2}$. Since $a$ and $b$ were arbitrary nonzero complex numbers, $A$ and $B$ are arbitrary positive real numbers.

\section{The Hermitian Counterpart of a Non-Hermitian Hamiltonian}

If for complex numbers $\alpha$ and $\beta$ we have $\alpha \neq 0, \beta \neq 0$, and $\alpha+\beta \neq 0$, then by Theorem 3, for the Hamiltonian $H_{\alpha, \beta}$, we have a family of metric operators defined by (17) and depending on two arbitrary positive real parameters $A$ and $B$. Each concrete choice of the metric operator $\eta_{+}$determines a Hermitian counterpart $h$ of $H_{\alpha, \beta}$. In this section, we will calculate $h$ in two particular cases.

(1) Assume that

$$
\beta=c \alpha,
$$

where $c$ is a positive real number.

Obviously, if $\eta_{+}$is a metric operator for $H_{\alpha, \beta}$, then any positive scalar multiple of $\eta_{+}$is also a metric operator for the same Hamiltonian $H_{\alpha, \beta}$. Therefore, dividing both sides of (17) by $A$ and setting $c=B / A$, we get, due to condition (60), that the operator $\eta_{+}$defined by

$$
\left(\eta_{+} \psi\right)(x)= \begin{cases}\psi(x) & \text { if } x \in[-1,0), \\ c|\alpha|^{2} \psi(x) & \text { if } x \in(0,1]\end{cases}
$$

is a metric operator for the Hamiltonian $H_{\alpha, c \alpha}$. The inner product determined by this metric operator has the form

$$
\langle\psi, \phi\rangle_{+}=\int_{-1}^{0} \psi(x) \bar{\phi}(x) d x+c|\alpha|^{2} \int_{0}^{1} \psi(x) \bar{\phi}(x) d x .
$$

It follows that the operator $\rho=\sqrt{\eta_{+}}$is given by

$$
(\rho \psi)(x)= \begin{cases}\psi(x) & \text { if } x \in[-1,0), \\ |\alpha| \sqrt{c} \psi(x) & \text { if } x \in(0,1]\end{cases}
$$

and its inverse $\rho^{-1}$ by

$$
\left(\rho^{-1} \psi\right)(x)= \begin{cases}\psi(x) & \text { if } x \in[-1,0), \\ \frac{1}{|\alpha| \sqrt{c}} \psi(x) & \text { if } x \in(0,1] .\end{cases}
$$

We can now calculate the operator

$$
h=\rho H_{\alpha, c \alpha} \rho^{-1} .
$$

The domain of definition $D(h)$ of the operator $h$ consists of all functions $\psi \in L^{2}(-1,1)$ such that $\rho^{-1} \psi \in D\left(H_{\alpha, c \alpha}\right)$. Therefore $D(h)$ consists of all functions $\psi \in W^{2,2}([-1,0) \cup(0,1])$ satisfying the transition conditions

$$
\psi\left(0^{-}\right)=\frac{\alpha}{|\alpha| \sqrt{c}} \psi\left(0^{+}\right), \quad \psi^{\prime}\left(0^{-}\right)=\frac{\alpha \sqrt{c}}{|\alpha|} \psi^{\prime}\left(0^{+}\right)
$$

and the boundary conditions

$$
\psi^{\prime}(-1)=\psi^{\prime}(1)=0 .
$$

Moreover, (65) implies by (63) and (64) that if $\psi \in D(h)$, then

$$
(h \psi)(x)=-\psi^{\prime \prime}(x), \quad x \in[-1,0) \cup(0,1] .
$$

We see that

$$
h=H_{z, w} \quad \text { with } z=\frac{\alpha}{|\alpha| \sqrt{c}}, w=\frac{\alpha \sqrt{c}}{|\alpha|} .
$$

Since $z \bar{w}=1, h$ is Hermitian by Theorem 1 .

(2) As a second example, consider the case where $\alpha$ and $\beta$ are arbitrary positive real numbers. By Theorem 1 , the Hamiltonian $H_{\alpha, \beta}$ is non-Hermitian if $\alpha \beta \neq 1$. Taking $B=$ $A \beta / \alpha$ in (17), we find that the operator $\eta_{+}$defined by

$$
\left(\eta_{+} \psi\right)(x)= \begin{cases}\psi(x) & \text { if } x \in[-1,0), \\ \alpha \beta \psi(x) & \text { if } x \in(0,1]\end{cases}
$$

is a metric operator for the Hamiltonian $H_{\alpha, \beta}$. The inner product determined by this metric operator has the form

$$
\langle\psi, \phi\rangle_{+}=\int_{-1}^{0} \psi(x) \bar{\phi}(x) d x+\alpha \beta \int_{0}^{1} \psi(x) \bar{\phi}(x) d x .
$$

Further reasoning as in the case of the previous example, we find that the operator

$$
h=H_{z, w} \quad \text { with } z=\sqrt{\frac{\alpha}{\beta}}, w=\sqrt{\frac{\beta}{\alpha}}
$$

is a Hermitian counterpart of the Hamiltonian $H_{\alpha, \beta}$.

\section{Conclusion}

An axiom of the classical quantum mechanics dictates that physical observables and in particular the Hamiltonian must be Hermitian (self-adjoint) because Hermiticity guarantees that the energy spectrum is real and that time evolution is unitary (probability-preserving). However, during the past two decades, it was understood that non-Hermitian Hamiltonians having real spectrum can also be used in the quantum mechanics. For this purpose, one should construct a new inner product (scalar product) with respect to which the Hamiltonian, non-Hermitian with respect to the original inner product of the Hilbert space, turns out to be Hermitian. In this paper, for a class of concrete non-Hermitian Hamiltonians with real spectrum, we have constructed explicitly such inner products. 


\section{References}

[1] F. G. Scholtz, H. B. Geyer, and F. J. W. Hahne, "Quasi-Hermitian operators in quantum mechanics and the variational principle," Annals of Physics, vol. 213, no. 1, pp. 74-101, 1992.

[2] C. M. Bender, "Making sense of non-Hermitian Hamiltonians," Reports on Progress in Physics, vol. 70, no. 6, pp. 947-1018, 2007.

[3] A. Mostafazadeh, "Pseudo-Hermitian representation of quantum mechanics," International Journal of Geometric Methods in Modern Physics, vol. 7, no. 7, pp. 1191-1306, 2010.

[4] P. Siegl, Quasi-Hermitian models [M.S. thesis], Faculty of Nuclear Sciences and Physical Engineering, CTU, Prague, Czech Republic, 2007-2008, http://physics.ffi.cvut.cz/publications/mf/2005/siegl_thesis.pdf.

[5] A. Mostafazadeh, "Metric operator in pseudo-Hermitian quantum mechanics and the imaginary cubic potential," Journal of Physics A, vol. 39, no. 32, pp. 10171-10188, 2006.

[6] D. Krejčiř́ík, H. Bíla, and M. Znojil, "Closed formula for the metric in the Hilbert space of a PT-symmetric model," Journal of Physics A, vol. 39, no. 32, pp. 10143-10153, 2006.

[7] D. Krejčiríík, "Calculation of the metric in the Hilbert space of a PT-symmetric model via the spectral theorem," Journal of Physics A, vol. 41, no. 24, Article ID 244012, 6 pages, 2008.

[8] D. Krejcirik, P. Siegl, and J. Zelezny, “' On the similarity of Sturm-Liouville operators with non-Hermitian boundary conditions to self-adjoint andnormal operators," http://arxiv.org/ abs/1108.4946.

[9] P. Siegl, "Supersymmetric quasi-Hermitian Hamiltonians with point interactions on a loop," Journal of Physics A, vol. 41, no. 24, Article ID 244025, 11 pages, 2008.

[10] M. Znojil, "The complete menu of eligible metrics for a family of toy Hamiltonians $H \neq H^{\dagger}$ withreal spectra," http://arxiv.org/ abs/0806.4295.

[11] E. Ergun, "A two-parameter family of non-Hermitian Hamiltonians with real spectrum," Journal of Physics A, vol. 43, no. 45, Article ID 455212, 16 pages, 2010.

[12] E. Ergun and M. Saglam, "On the metric of a non-Hermitian model," Reports on Mathematical Physics, vol. 65, no. 3, pp. 367$378,2010$.

[13] I. C. Gohberg and M. G. Krein, Introduction to the Theory of Linear Nonselfadjoint Operators, vol. 8 of Translations of Mathematical Monographs, American Mathematical Society, Providence, RI, USA, 1969. 


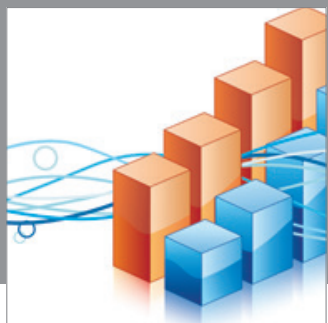

Advances in

Operations Research

mansans

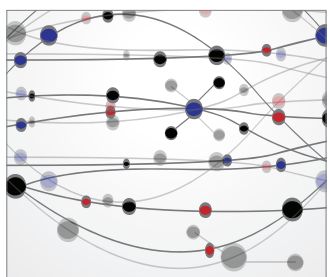

The Scientific World Journal
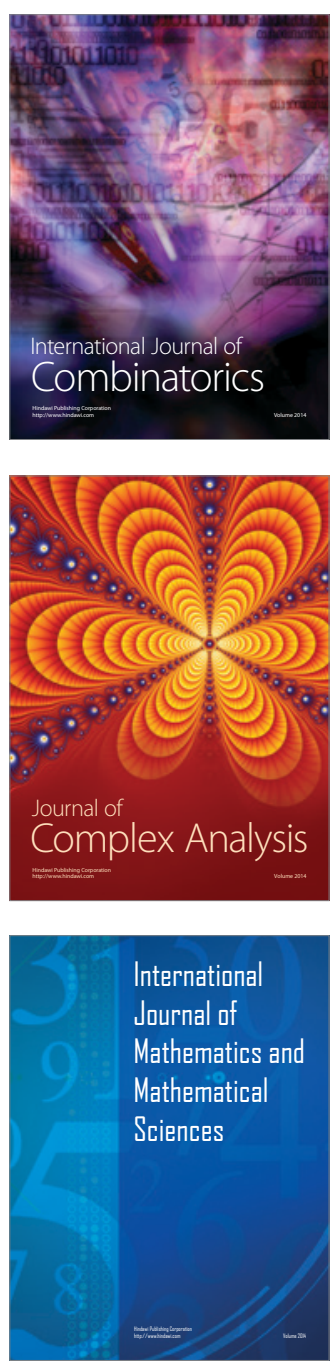
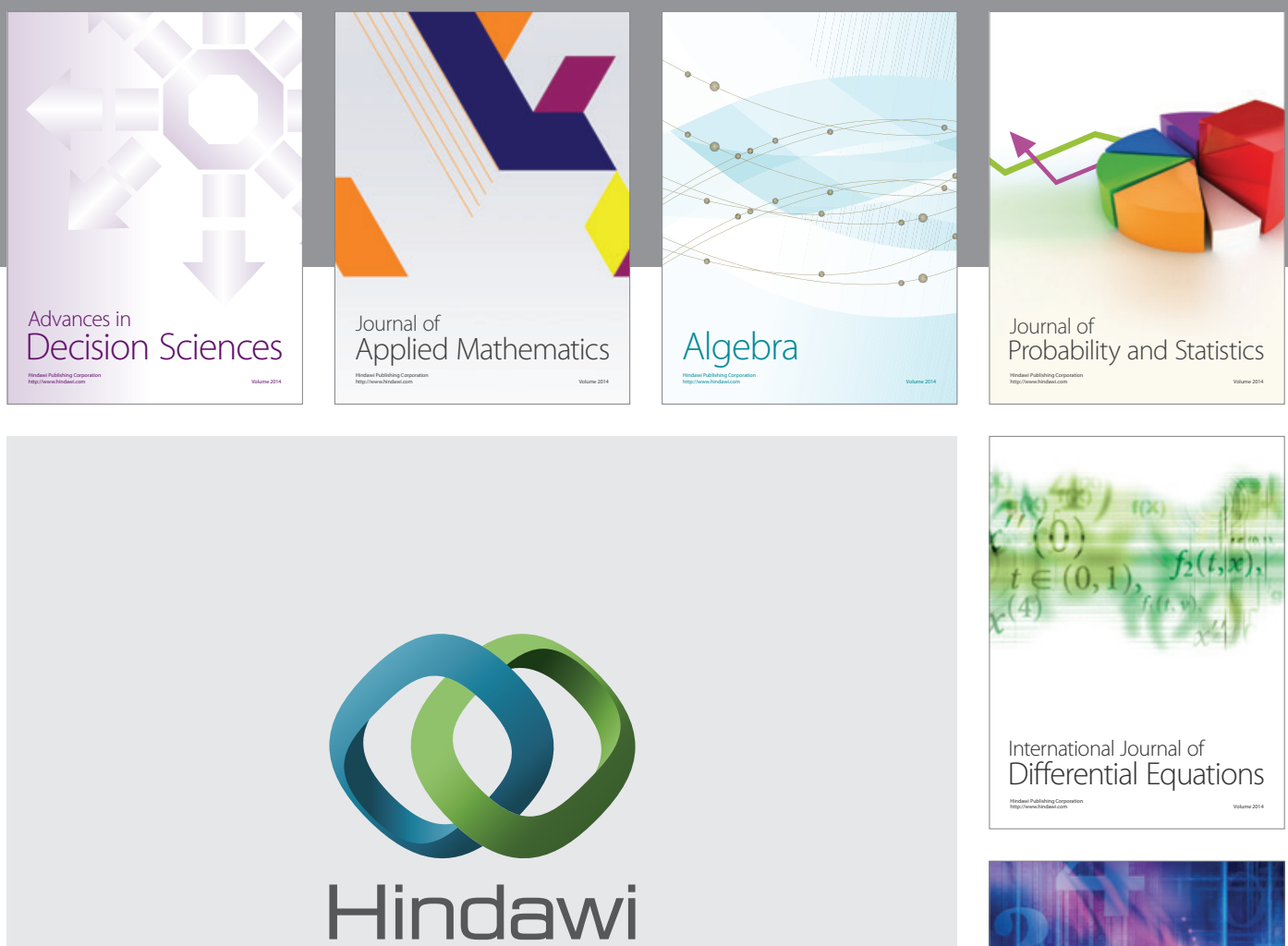

Submit your manuscripts at http://www.hindawi.com
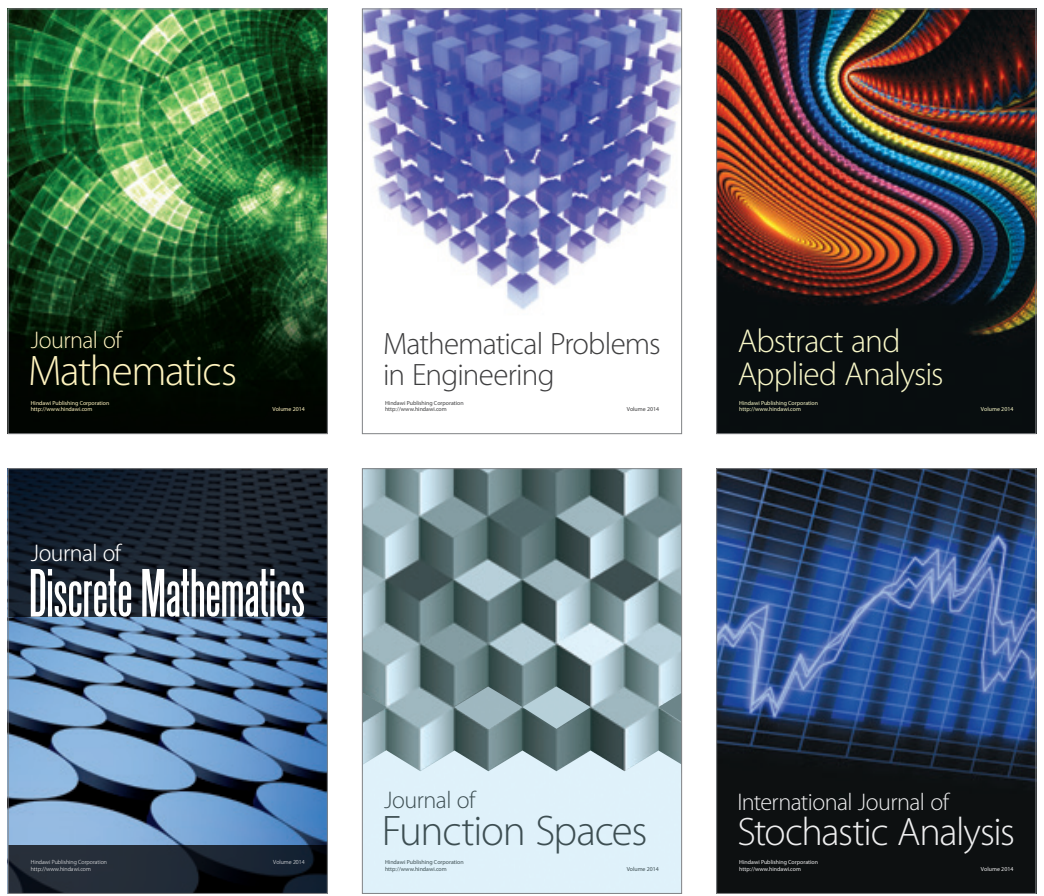

Journal of

Function Spaces

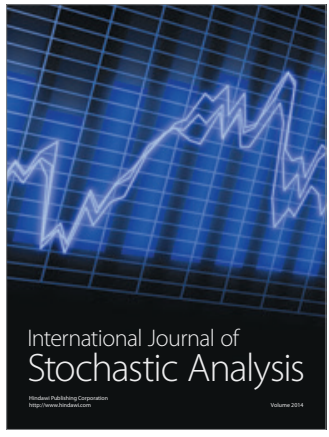

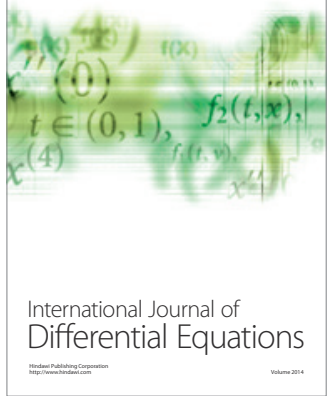
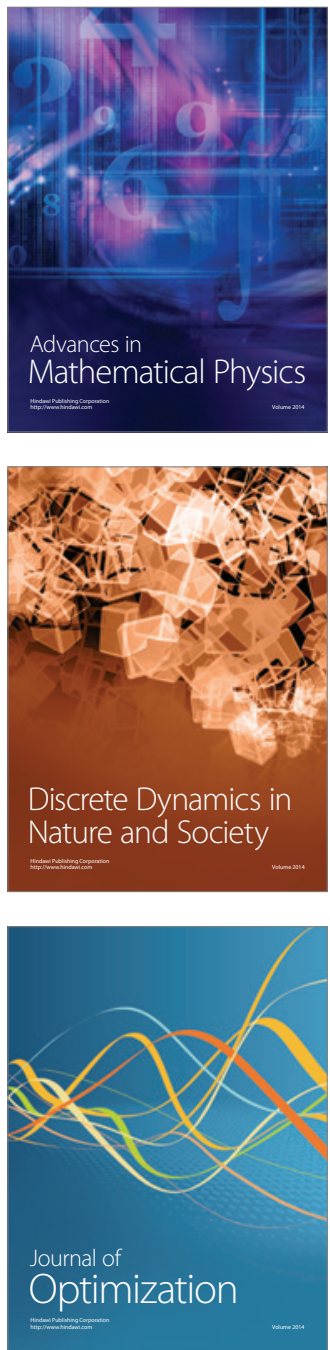